\title{
Estimation and evaluation of COSMIC radio occultation excess phase using undifferenced measurements
}

\author{
Pengfei Xia, Shirong Ye, Kecai Jiang, and Dezhong Chen \\ GNSS Research Centre, Wuhan University, Wuhan, 430079, China \\ Correspondence to: Shirong Ye (srye@whu.edu.cn)
}

Received: 23 August 2016 - Discussion started: 16 November 2016

Revised: 20 April 2017 - Accepted: 23 April 2017 - Published: 16 May 2017

\begin{abstract}
In the GPS radio occultation technique, the atmospheric excess phase (AEP) can be used to derive the refractivity, which is an important quantity in numerical weather prediction. The AEP is conventionally estimated based on GPS double-difference or single-difference techniques. These two techniques, however, rely on the reference data in the data processing, increasing the complexity of computation. In this study, an undifferenced (ND) processing strategy is proposed to estimate the AEP. To begin with, we use PANDA (Positioning and Navigation Data Analyst) software to perform the precise orbit determination (POD) for the purpose of acquiring the position and velocity of the mass centre of the COSMIC (The Constellation Observing System for Meteorology, Ionosphere and Climate) satellites and the corresponding receiver clock offset. The bending angles, refractivity and dry temperature profiles are derived from the estimated AEP using Radio Occultation Processing Package (ROPP) software. The ND method is validated by the COSMIC products in typical rising and setting occultation events. Results indicate that rms (root mean square) errors of relative refractivity differences between undifferenced and atmospheric profiles (atmPrf) provided by UCAR/CDAAC (University Corporation for Atmospheric Research/COSMIC Data Analysis and Archive Centre) are better than 4 and $3 \%$ in rising and setting occultation events respectively. In addition, we also compare the relative refractivity bias between ND-derived methods and atmPrf profiles of globally distributed 200 COSMIC occultation events on 12 December 2013. The statistical results indicate that the average rms relative refractivity deviation between ND-derived and COSMIC profiles is better than $2 \%$ in the rising occultation event and better than $1.7 \%$ in the setting occultation event. Moreover, the observed COSMIC refrac-
\end{abstract}

tivity profiles from ND processing strategy are further validated using European Centre for Medium-Range Weather Forecasts (ECMWF) analysis data, and the results indicate that the undifferenced method reduces the noise level on the excess phase paths in the lower troposphere compared to the single-difference processing strategy.

\section{Introduction}

The radio occultation (RO) technique was first applied in the field of astronomy to detect the state of the planet's atmosphere (Kursinski et al., 1997). With the development of GPS meteorology, the space-based GPS radio occultation is regarded as a valuable tool for atmospheric change studies (Rocken et al., 1997; Kursinski et al., 1997; Hajj et al., 2002; Beyerle et al., 2005). Since the GPS/MET (Global Positioning System/Meteorology) mission has conducted a number of successful measurement experiments from 1995 to 1997 (Ware, 1996; Rocken et al., 1997), some low Earthorbiting (LEO) satellites, such as CHAMP, GRACE, COSMIC (The Constellation Observing System for Meteorology, Ionosphere and Climate) and MetOp-A (Wickert et al., 2001, 2005; Rocken et al., 2000; Wilson et al., 2010) have begun to equip RO instruments to facilitate the development of the RO technique. In the GPS occultation technique, the atmospheric refractivity is an important quality in numerical weather prediction (Esteban et al., 2013). The atmospheric excess phase (AEP) can be used to derive the bending angles of the GPS rays as well as to obtain the refractivity from the bending angles. Thus, the retrieval accuracy of refractivity is quite dependent on the quality of the estimated AEP. 
Conventionally, the AEP is determined using two kinds of differential techniques, i.e. double-difference (DD; Sokolovskiy and Hunt, 1996; Kursinski et al., 1997; Rocken et al., 1997; Hajj et al., 2002) and single-difference (SD; Wickert et al., 2002; Schreiner et al., 2005), in which various errors can be eliminated from the differencing operation of GPS observations. The double-difference method requires additional data from the ground receiver as well as the GPS reference satellite to remove the oscillator errors of the transmitter and the LEO receiver. This processing will bring additional error sources to AEP from the ground data and the GPS reference satellite, such as multipath error, residual ionospheric and troposphere errors, thermal noise and so on (Schreiner and Rocken, 2010). Single-difference processing has a potential advantage over double-differencing strategy since it can eliminate the ground observation data error (Schreiner et al., 2010). However, single-difference excess phases also suffer the effect of the noise sources from the reference link data. Compared with the two differential techniques, the non-difference (ND) method does not require the reference link data, which reduces the complexity in data processing. Besides, the ND processing can potentially obtain AEPs with lower noise by utilizing previously estimated LEO and GPS clocks (Beyerle et al., 2005; Schreiner et al., 2011). Beyerle et al. (2005) firstly proposed the idea of using an ND technique to estimate the AEP and successfully analysed the GRACE-B satellite, correcting for the effect of receiver clock by interpolating the temporal resolution of $30 \mathrm{~s}$ GRACE-B's receiver clock solutions into $20 \mathrm{~ms}$. Their results show that there is a good agreement of the refractivity between ND and SD techniques in the upper troposphere and lower stratosphere. More significantly, the ND technique can reduce the noise level and yield less-biased refractivity in the lower troposphere compared to the SD technique. However, the ND technique needs a LEO receiver with an ultra-stable oscillator (Beyerle et al., 2005; Schreiner et al., 2010). Therefore, the single-difference method is still widely utilized to get the AEP in various GPS RO data processing centres (Bi et al., 2012).

The accuracy of the ND-derived refractivity mainly depends on the quality of the receiver clock. COSMIC satellites' orbits and clock solutions are provided at a temporal resolution of $30 \mathrm{~s}$ (CDAAC, 2013). Due to the effect of COSMIC receiver oscillator, a lot of noise will be introduced when interpolating $30 \mathrm{~s}$ clock solutions into $20 \mathrm{~ms}$. In this study, we adopt PANDA (Positioning And Navigation Data Analyst) software to determine the COSMIC satellite orbit and obtain the receiver clock offset at an interval of 1s (Liu and Ge, 2003). Then, the AEP is extracted by utilizing the ND technique. Additionally, the refractivity and dry temperature will be derived from the AEP based on the ROPP (Radio Occultation Processing Package) software. Finally, we compare the ND-derived refractivity with the atmPrf profiles provided by UCAR/CDAAC (University Corporation for Atmospheric Research/COSMIC Data Analysis and Archive
Centre). Moreover, the ND-derived refractivity profiles are further evaluated by comparing them with the field data by the ECMWF (European Centre for Medium-Range Weather Forecasts).

The rest of the paper is organized as follows. Section 2 introduces the principle of estimating AEP using a nondifference method. Section 3 describes the processing of the LEO precise orbit determination using the PANDA software. Section 4 presents the validation of the ND-derived method. The conclusions are included in Sect. 5.

\section{Non-differencing method}

The signals of the GPS occulting satellites are recorded by the RO receiver aboard the spacecraft at $50 \mathrm{~Hz}$ during the occultation event. The carrier phase measurements with repaired cycle slips can be expressed as follows (Schreiner et al., 2010):

$$
\begin{aligned}
L i_{r}^{s}\left(t_{\mathrm{r}}\right) & =c \cdot \delta t_{\mathrm{r}}\left(t_{\mathrm{r}}\right) \\
& +c \cdot \delta t_{\mathrm{r}, \mathrm{rel}}\left(t_{\mathrm{r}}\right)+\rho_{r}^{s}\left(t_{\mathrm{r}}\right)+\rho_{\mathrm{r}, \text { rel }}^{s}\left(t_{\mathrm{r}}\right)+c \cdot \delta t^{\mathrm{s}}\left(t_{\mathrm{r}}-\tau_{r}^{s}\right) \\
& +c \cdot \delta t_{\mathrm{rel}}\left(t_{\mathrm{r}}-\tau_{r}^{s}\right)+\delta \rho_{\mathrm{r}, \text { ion }}^{s}\left(t_{\mathrm{r}}\right)+\delta \rho_{\mathrm{r}, \text { trop }}^{s}\left(t_{\mathrm{r}}\right) \\
& +\lambda_{i} \cdot N_{\mathrm{amb}}+V_{\mathrm{pco}}+\varepsilon
\end{aligned}
$$

where $i=1,2 ; t_{\mathrm{r}}$ and $\delta t_{\mathrm{r}}$ denote the receiving time and the deviation between receiver time and system time at the observing epoch, respectively; $c$ is the speed of light in a vacuum; $\delta t_{\mathrm{r}, \text { rel }}$ represents the offset between right time and coordinate time at the receiver owing to special and general relativity; $t^{\mathrm{s}}$ and $\delta t^{\mathrm{s}}$ denote the transmitted time and the deviation between proper time and satellite time at transmit time respectively; $\delta t_{\text {rel }}^{\mathrm{s}}$ expresses the offset between the right time and the coordinate time at the satellite; $\rho_{r}^{\mathrm{s}}$ is the geometric range between the GPS satellite and COSMIC satellite; $\delta \rho_{\mathrm{r} \text {,rel }}^{\mathrm{s}}$ is gravitational delay correction; $\delta \rho_{r \text {,ion }}^{\mathrm{s}}$ and $\delta \rho_{\mathrm{r} \text {,trop }}^{\mathrm{s}}$ signify the ionospheric delay correction and tropospheric delay correction respectively; $\tau_{t}^{\mathrm{s}}$ indicates the light travel time in vacuum; $N_{\text {amb }}$ represents phase ambiguity; $V_{\text {pco }}$ is the antenna phase centre offsets; $\varepsilon$ is the carrier phase measurement noise.

The above equation neglects multipath errors, carrier phase wind-up and so on. The orbit and the clock offsets of GPS satellites are provided by the International GNSS Service (IGS). In addition, the $\tau_{t}^{\mathrm{s}}, \delta t_{\text {rel }}^{\mathrm{s}}$ and $\delta \rho_{\mathrm{r}, \text { rel }}^{\mathrm{s}}$ can be modelled as follows (Schreiner et al., 2010):

$$
\begin{aligned}
& \tau_{r}^{s}=\frac{\left(\rho_{r}^{s}\left(t_{\mathrm{r}}\right)+\delta \rho_{\mathrm{r}, \mathrm{rel}}^{s}\right)}{c} \\
& \delta t_{\mathrm{rel}}^{s}=-2 \frac{r^{s} \cdot v^{s}}{c^{2}} \\
& \delta \rho_{\mathrm{r}, \text { rel }}^{s}=\frac{2 G M_{\mathrm{E}}}{c^{2}} \ln \left(\frac{r^{S}+r_{\mathrm{r}}+\rho_{r}^{s}}{r^{S}+r_{\mathrm{r}}-\rho_{r}^{s}}\right),
\end{aligned}
$$

where $r^{\mathrm{s}}$ and $r_{\mathrm{r}}$ are the position and velocity vectors of the GPS satellite at signal transmit time in an Earth-centred in- 
Table 1. The rms of COSMIC satellites compared with UCAR/CDAAC on three axes.

\begin{tabular}{lrr|rr|rr|rr}
\hline & \multicolumn{2}{c|}{$\begin{array}{c}\text { Radial } \\
\text { rms }\end{array}$} & \multicolumn{2}{c|}{$\begin{array}{c}\text { Along-track } \\
\text { rms }\end{array}$} & \multicolumn{2}{c|}{$\begin{array}{c}\text { Cross-track } \\
\text { rms }\end{array}$} & \multicolumn{2}{c}{$\begin{array}{c}\text { 3-D Root } \\
\text { sum square }\end{array}$} \\
\cline { 2 - 9 } & $\begin{array}{r}\text { Pos } \\
(\mathrm{cm})\end{array}$ & $\begin{array}{r}\text { Vel } \\
\left(\mathrm{mm} \mathrm{s}^{-1}\right)\end{array}$ & $\begin{array}{r}\text { Pos } \\
(\mathrm{cm})\end{array}$ & $\begin{array}{r}\text { Vel } \\
\left(\mathrm{mm} \mathrm{s}^{-1}\right)\end{array}$ & $\begin{array}{r}\text { Pos } \\
(\mathrm{cm})\end{array}$ & $\begin{array}{r}\text { Vel } \\
\left(\mathrm{mm} \mathrm{s}^{-1}\right)\end{array}$ & $\begin{array}{r}\text { Pos } \\
(\mathrm{cm})\end{array}$ & $\begin{array}{r}\text { Vel } \\
\left(\mathrm{mm} \mathrm{s}^{-1}\right)\end{array}$ \\
\hline FM1 & 10.82 & 0.117 & 13.29 & 0.115 & 13.91 & 0.134 & 22.07 & 0.212 \\
FM2 & 10.40 & 0.162 & 16.99 & 0.105 & 9.65 & 0.097 & 22.13 & 0.216 \\
FM4 & 7.92 & 0.103 & 12.38 & 0.085 & 11.70 & 0.120 & 18.78 & 0.180 \\
FM5 & 8.89 & 0.123 & 14.39 & 0.097 & 12.48 & 0.087 & 21.01 & 0.179 \\
FM6 & 10.06 & 0.139 & 16.28 & 0.109 & 10.63 & 0.094 & 21.89 & 0.200 \\
Mean & 9.62 & 0.129 & 14.66 & 0.102 & 11.67 & 0.106 & 21.18 & 0.197 \\
\hline
\end{tabular}

Table 2. The rms of COSMIC satellites compared with overlapping orbits on three axes.

\begin{tabular}{lrr|rr|rr|rr}
\hline & \multicolumn{2}{c|}{$\begin{array}{c}\text { Radial } \\
\text { rms }\end{array}$} & \multicolumn{2}{c|}{$\begin{array}{c}\text { Along-track } \\
\text { rms }\end{array}$} & \multicolumn{2}{c|}{$\begin{array}{c}\text { Cross-track } \\
\text { rms }\end{array}$} & \multicolumn{2}{c}{$\begin{array}{c}\text { 3-D root } \\
\text { sum square }\end{array}$} \\
\cline { 2 - 9 } & $\begin{array}{r}\text { Pos } \\
(\mathrm{cm})\end{array}$ & $\begin{array}{r}\text { Vel } \\
\left(\mathrm{mm} \mathrm{s}^{-1}\right)\end{array}$ & $\begin{array}{r}\text { Pos } \\
(\mathrm{cm})\end{array}$ & $\begin{array}{r}\text { Vel } \\
\left(\mathrm{mm} \mathrm{s}^{-1}\right)\end{array}$ & $\begin{array}{r}\text { Pos } \\
(\mathrm{cm})\end{array}$ & $\begin{array}{r}\text { Vel } \\
\left(\mathrm{mm} \mathrm{s}^{-1}\right)\end{array}$ & $\begin{array}{r}\text { Pos } \\
(\mathrm{cm})\end{array}$ & $\begin{array}{r}\text { Vel } \\
\left(\mathrm{mm} \mathrm{s}^{-1}\right)\end{array}$ \\
\hline FM1 & 5.37 & 0.051 & 6.57 & 0.058 & 6.67 & 0.048 & 10.80 & 0.091 \\
FM2 & 5.30 & 0.058 & 6.55 & 0.054 & 5.63 & 0.049 & 10.13 & 0.093 \\
FM4 & 6.11 & 0.079 & 8.09 & 0.058 & 6.30 & 0.056 & 11.93 & 0.113 \\
FM5 & 4.94 & 0.069 & 8.07 & 0.053 & 7.02 & 0.057 & 11.78 & 0.105 \\
FM6 & 6.91 & 0.079 & 8.58 & 0.077 & 7.35 & 0.057 & 13.25 & 0.124 \\
Mean & 5.73 & 0.067 & 7.57 & 0.060 & 6.59 & 0.053 & 11.57 & 0.105 \\
\hline
\end{tabular}

ertial (ECI) reference frame (Ashby, 2003); $G$ denotes Newton's gravitational constant; $M_{\mathrm{E}}$ expresses the Earth's mass; $r^{\mathrm{s}}$ and $r_{\mathrm{r}}$ represent the GPS satellite and receiver radial positions at the GPS signal transmit and receive times.

The L1 and L2 channel phases can be combined with satellite position and velocity data to determine the AEP. By neglecting the influences of ambiguity and time-independent error terms, the use of the ND method to calculate the AEP $\left(\Delta L_{i}\right)$ can be modelled as follows (Schreiner et al., 2010):

$$
\begin{aligned}
\Delta L_{i} & =\delta \rho_{\mathrm{r}, \text { ion }}^{s}\left(t_{\mathrm{r}}\right)+\delta \rho_{\mathrm{r}, \text { trop }}^{s}\left(t_{\mathrm{r}}\right) \\
& =L i_{r}^{s}\left(t_{\mathrm{r}}\right)-c \cdot \delta t_{\mathrm{r}}\left(t_{\mathrm{r}}\right)-c \cdot \delta t_{\mathrm{r}, \mathrm{rel}}\left(t_{\mathrm{r}}\right)-\rho_{r}^{s}\left(t_{\mathrm{r}}\right) \\
& -\rho_{\mathrm{r}, \mathrm{rel}}^{s}\left(t_{\mathrm{r}}\right)-c \cdot \delta t^{\mathrm{s}}\left(t_{\mathrm{r}}-\tau_{r}^{s}\right) \\
& -c \cdot \delta t_{\mathrm{rel}}\left(t_{\mathrm{r}}-\tau_{r}^{s}\right)-V_{\mathrm{pco}}
\end{aligned}
$$

The input L1 and L2 phase measures of COSMIC RO are provided by the opnGps profiles which can be provided by the UCAR/CDAAC at a temporal resolution of $20 \mathrm{~ms}$. Besides, UCAR/CDAAC also supplies the COSMIC receiver clock offset leoClk profile and the GPS satellite clock offset comClk profile at a temporal resolution of $30 \mathrm{~s}$. Each COSMIC satellite is equipped with the BlackJack GPS receiver, a tiny ionospheric photometer (TIP) and a tri-band beacon (TBB; Wu et al., 2005; Schreiner, 2005; Montenbruck et al., 2006), and the Integrated GPS Occultation Receiver (IGOR) is designed by the Jet Propulsion Laboratory (JPL) and man- ufactured by Broad Reach Engineering (Schreiner et al., 2011). By analysing the results of leoClk profiles, it can be concluded that the vibration of the IGOR receiver clock is not sufficiently stable. Therefore, estimated AEP at the required temporal resolution of $20 \mathrm{~ms}$ could not be interpolated successfully from the 30 s clock solutions when utilizing Eq. (5). A technique can be effective at dealing with this problem by reprocessing the COSMIC satellite orbit and obtaining a high-accuracy and high-temporal resolution IGOR clock offset.

\section{COSMIC spacecraft precise orbit determination}

\subsection{COSMIC POD processing}

The joint Taiwan/US mission COSMIC, which includes six micro-satellites, was launched on 17 April 2006. Each satellite is equipped with a GPS receiver, which is installed with four antennas on the front and back faces of the satellite main frame. Two single-patch antennas, mounted on the upper part of the main body, are used for the purpose of POD. The other two antennas, dedicated to atmospheric occultation research, are mounted on the lower part (Hwang et al., 2009). The POD of the COSMIC satellite is an important premise in atmospheric occultation research. At present, UCAR (University Corporation for Atmospheric Research) provides three kinds 

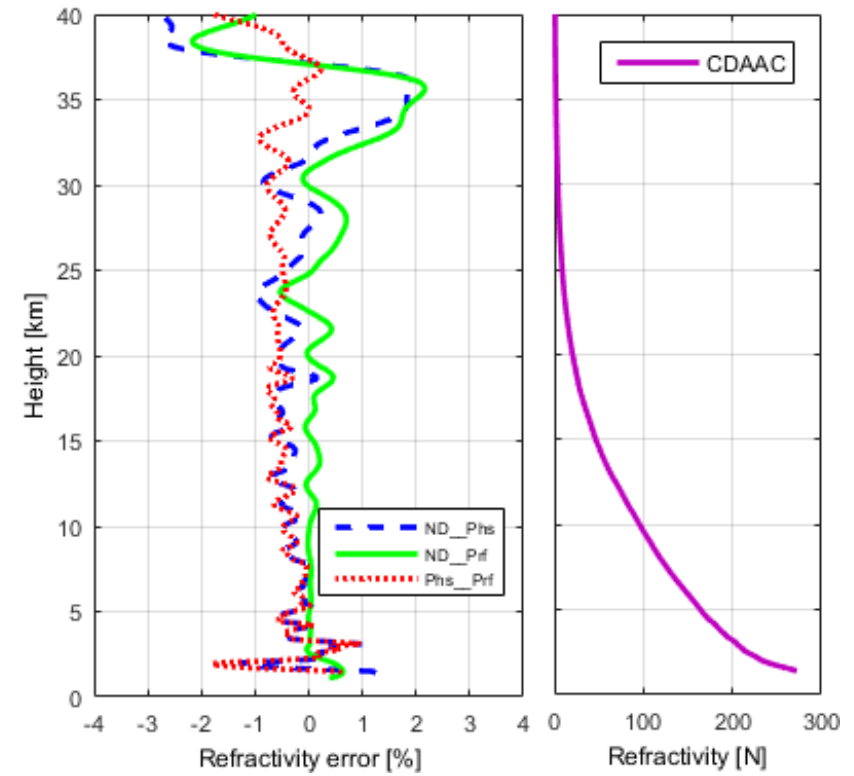

Figure 1. The results of ND_Phs, ND_Prf and Phs_Prf in the setting radio occultation. ND_Phs expresses the relative refractivity offset between Ref_ND and Ref_Phs; ND_Prf denotes the relative refractivity offset between Ref_ND and atmPrf; Phs_Prf indicates the relative refractivity offset between Ref_Phs and atmPrf products; Ref_ND is the refractivity obtained from ND-derived AEP based on ROPP software; Ref_Phs is the refractivity obtained from atmPhs profiles based on ROPP software.

of COSMIC orbit products, i.e. reprocessing products, postproducts and real-time products. UCAR/CDAAC reprocessing products adopt Bernese 5.2 software as a processing tool, and orbit determination method is improved and the processing method of the phase values is more detailed. The average rms value of three-dimensional overlapping orbit precision is superior to $15 \mathrm{~cm}$, and the three-dimensional velocity $\mathrm{rms}$ value is better than $0.15 \mathrm{~mm} \mathrm{~s}^{-1}$ (CDAAC, 2013). In addition, Hwang et al. (2009) calculated the COSMIC satellite orbits using Bernese 5.0 software and differences between their orbit products and those of UCAR are at the level of $10 \mathrm{~cm}$ on three axes.

PANDA is satellite positioning and orbit determination software which is developed by satellite navigation and positioning technology research centre of Wuhan University. The software has the ability to process a variety of observation data, such as GNSS (including GPS, GLONASS, GALILEO, and BDS), SLR (satellite laser ranging), KBR (K/Ka-Band Ranging), satellite attitude and so on (Liu et al., 2004). In the paper, the PANDA software is exploited to perform POD for the COSMIC satellite. These inputs include the COSMIC L1 and L2 pseudo-range and carrier phase data from the HAICH-FARR antenna, Centre for CODE (Centre for Orbit Determination in Europe) final GPS orbits, $5 \mathrm{~s}$ CODEprovided transmitter clock offsets in GPS time, LEO attitude information from CDAAC, Earth orientation information and
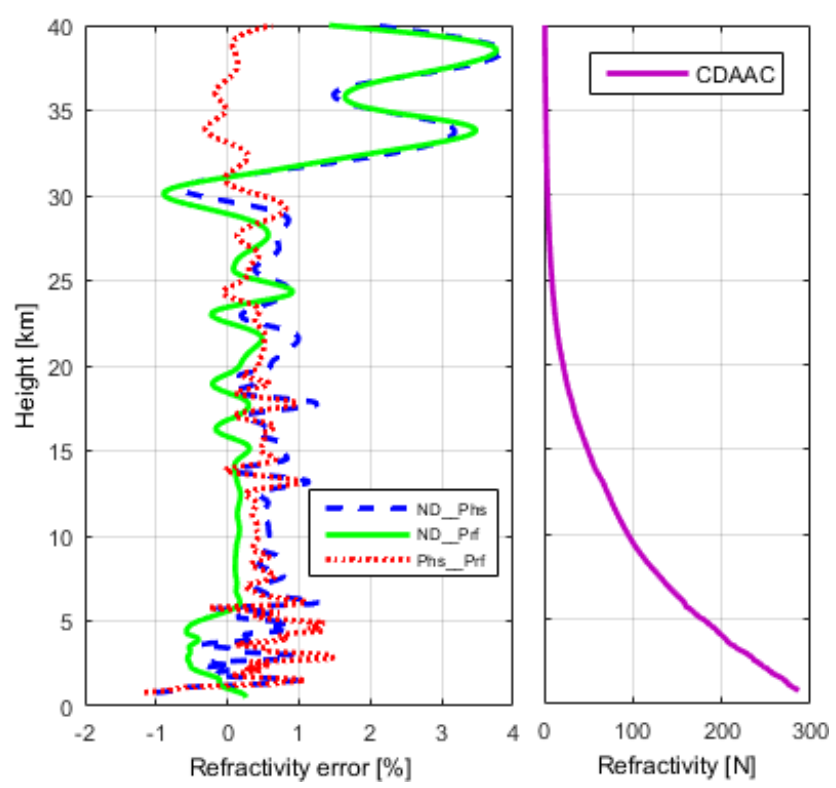

Figure 2. The results of ND_Phs, ND_Prf and Phs_Prf in the rising radio occultation. ND_Phs expresses the relative refractivity offset between Ref_ND and Ref_Phs; ND_Prf denotes the relative refractivity offset between Ref_ND and atmPrf; Phs_Prf indicates the relative refractivity offset between Ref_Phs and atmPrf products; Ref_ND is the refractivity obtained from ND-derived AEP based on ROPP software; Ref_Phs is the refractivity obtained from atmPhs profiles based on ROPP software.

L1 and L2 antenna phase centre variations. Ionosphere-free phase observations are utilized, based on a post-processed generalized least squares approach, to determine the position and velocity of the LEO satellite mass centre as a function of coordinate time in an ECI reference frame and the LEO clock every $1 \mathrm{~s}$. In this process, the POD is calculated over $30 \mathrm{~h}$ data arcs by utilizing $1 \mathrm{~Hz}$ carrier phase observations from HAICH-FARR antenna. The state vector computed in this process also takes the influence of the dynamic model or estimation method into account, such as the gravitational field, the Earth and ocean tides, tidal dynamic model, solar radiation pressure, empirical acceleration and so on. Furthermore, the gravity field uses EIGEN2 model with an order set to 140 , and the solar radiation pressure uses a box-wing model.

\subsection{POD precision evaluation}

In the processing of POD, we can first obtain the initial COSMIC satellite orbit, state parameters and mechanical parameters through pseudo-range single point positioning, then these initial solutions can be used for orbit integration for the purpose of further elaboration. The next stage of processing is to detect gross errors and cycle slips of the GPS carrier phase data, and then the COSMIC orbit precision will be improved according to the residual errors using an iter- 
Table 3. Details of parameters of the two selected GPS occultation events on 12 December 2013.

\begin{tabular}{lll}
\hline Parameter & OCCsat-1 & OCCsat-2 \\
\hline GPS & PRN-08 & PRN-28 \\
LEO & CO06 & CO05 \\
Start time & $15: 07$ & $14: 12$ \\
Duration & $123 \mathrm{~s}$ & $146 \mathrm{~s}$ \\
Status & Setting & Rising \\
Longitude & $3.78^{\circ} \mathrm{E}$ & $-109.84^{\circ} \mathrm{W}$ \\
Latitude & $34.06^{\circ} \mathrm{N}$ & $26.08^{\circ} \mathrm{N}$ \\
Quality mark & bad $=0$ & bad $=0$ \\
\hline
\end{tabular}

ative least squares estimate and residual edit operation. Finally, the orbit and clock error will be output every second. In order to test the accuracy of COSMIC orbits from the PANDA software, we select 53-day satellite-borne GPS observation data from day of year (DOY) 313 to 365 of 2011 which are provided by UCAR/CDAAC. Due to the missing of 3rd COSMIC satellite data, we only process the remaining five satellite data sets and remove those data set with observation time less than $10 \mathrm{~h}$. Since the COSMIC satellites are not equipped with laser corner reflector, the accuracy of orbit determination cannot be evaluated by SLR precision ranging information. Thus, we mainly compare our orbit results with UCAR/CDAAC to analyse the precision of orbit determination. The statistical results of five satellites orbit between those derived by PANDA and those derived by UCAR/CDAAC are given in Table 1. In addition, there is a $6 \mathrm{~h}$ overlapping orbit between the tracks because of the usage of $30 \mathrm{~h}$ data arcs as the orbit determination length. Table 2 presents the rms of COSMIC satellites, which are compared with overlapping orbits on three axis directions.

As shown in Table 1, the averages of 3-D rms orbit coordinates and velocity differences between PANDA and UCAR/CDAAC for the 53-day period are $21.18 \mathrm{~cm}$ and $0.20 \mathrm{~mm} \mathrm{~s}^{-1}$ respectively. In addition, Table 1 shows that the accuracy of the POD from the 4th COSMIC satellite is better than from the other four satellites. Table 2 shows the average 3-D rms POD coordinates and velocity are better than $11.57 \mathrm{~cm}$ and $0.11 \mathrm{~mm} \mathrm{~s}^{-1}$ when compared with overlapping orbits and velocity, respectively. These results suggest that the COSMIC POD generated from PANDA software agrees well with the UCAR/CDAAC-derived orbit information.

\section{Result validation and analysis}

The UCAR/CDAAC AEP products for COSMIC are generated by a single-difference processing approach and deposited into an atmPhs profile. Atmospheric profiles of bending angle, refractivity and dry temperature generated from atmPhs profiles were written into atmPrf profiles. These profiles are publicly available at http://cdaac-www.cosmic.ucar.

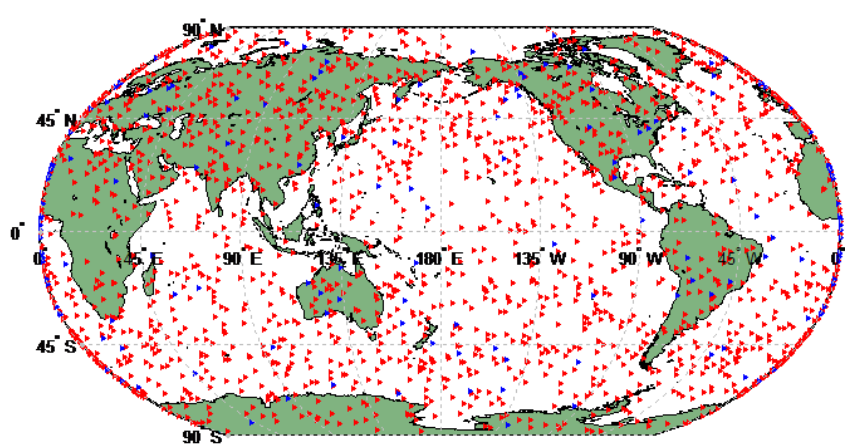

Figure 3. The global distribution of COSMIC RO events on $12 \mathrm{De}-$ cember 2013. Blue triangle represents the selected $200 \mathrm{RO}$ events.

Table 4. Summary of the comparison between ROPP-derived and atmPrf. ND-phs represents the comparison between ND-derived and atmPhs-derived; ND-prf represents the comparison between ND-derived and atmPrf; phs-prf represents the comparison between atmPhs-derived and atmPrf (\%).

\begin{tabular}{llrrr}
\hline Parameter & Status & ND-phs & ND-prf & phs-prf \\
\hline Refractivity & rising & 1.64 & 1.91 & 0.93 \\
& setting & 1.52 & 1.63 & 0.51 \\
Dry & rising & 2.49 & 3.21 & 1.65 \\
Temperature & setting & 2.35 & 2.42 & 0.72 \\
\hline
\end{tabular}

edu/. In order to evaluate the precision of AEP estimated by the ND method, the AEPs are obtained using Eq. (5) in this study. Then, the ROPP software is implemented to process excess phase data and derive profiles of bending angle, dry temperature and refractivity. Finally, the obtained COSMIC refractivity profiles are compared with atmPrf profiles provided by UCAR/CDAAC. Furthermore, UCAR/CDAAC offers the moisture profiles generated from ECMWF analysis and the ERA-Interim model which collocated with occultation profiles. So the comparison between ND-derived refractivity and meteorological analysis results are performed to further validate the results obtained from ND method. We use the same transmitter-receiver pair to compare the collocated measurements and retrievals with UCAR/CDAAS.

\subsection{The results of a typical GPS occultation event}

A non-difference processing strategy is utilized to obtain L1 and L2 excess atmospheric phases as functions of GPS time in an ECI TOD (true equator and equinox of data) reference frame. Inputs to this processing are $50 \mathrm{~Hz} \mathrm{L1}$ and $\mathrm{L} 2$ phase measures for the occulting GPS satellite, LEO and GPS positions, velocities and clock offsets, and antenna phase centre information. Then, the AEPs are calculated using Eq. (5), randomly selecting two GPS occultation events on 12 December 2013. Table 3 gives details of the parameters from the two GPS occultation events. 


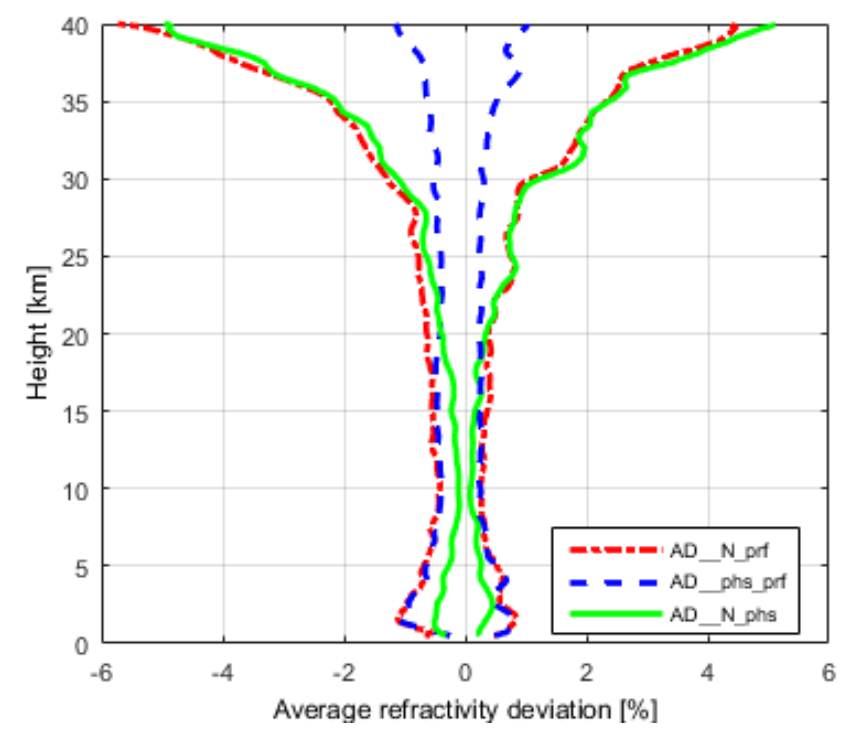

Figure 4. The statistical average relative deviation results of the refractivity for setting occultation events. AD_N_prf denotes the average relative deviation of refractivity between $\mathrm{R} \_\mathrm{N}$ and atmPrf; AD_phs_prf expresses the average relative deviation of refractivity between R_phs and atmPrf; AD_N_phs represents average relative deviation of refractivity between $\mathrm{R} \_N$ and $\mathrm{R} \_$phs; $\mathrm{R} \_\mathrm{N}$ is the refractivity derived from ND-derived AEP for the selected 200 RO events based on ROPP software; Ref_Phs is the refractivity obtained from atmPhs profiles for the selected $200 \mathrm{RO}$ events based on ROPP software.

As with the method introduced in Sect. 2, we first process the two GPS occultation events to obtain AEPs using the ND technique. Afterwards, these AEPs and collocated occultation atmPhs profiles will be used to generate refractivity based on the ROPP software. We name them Ref_ND and Ref_Phs. Using the atmPrf profiles of refractivity provided by UACR/CDAAC as references, the ROPP software is validated by comparing Ref_Phs with atmPrf products. Moreover, the Ref_ND is evaluated by comparing it with the Ref_Phs and atmPrf products. These results are depicted by Figs. 1 and 2.

Figures 1 and 2 show the results for the setting and rising occultations respectively. Phs_Prf is less than $\pm 1.7 \%$ below $40 \mathrm{~km}$, which verifies the feasibility of ROPP software. In addition, ND_Prf is closer to 0 than Phs_Prf and ND_Phs, which are below $30 \mathrm{~km}$ while Phs_Prf and ND_Phs gradually increase from 30 to $40 \mathrm{~km}$. Besides, Figs. 1 and 2 also reveal that the refractivity changes exponentially with height, and the refractivity is less than $5 \mathrm{~N}$ from 30 to $40 \mathrm{~km}$.

\subsection{Statistics and verification of the ND method}

The COSMIC RO provides about $1800 \mathrm{RO}$ events per day and its scientific mission is mainly for weather, climate, space weather, geodetic research and other study purposes (Yen et al., 2007; Kuo et al., 2007). To verify the ND method,

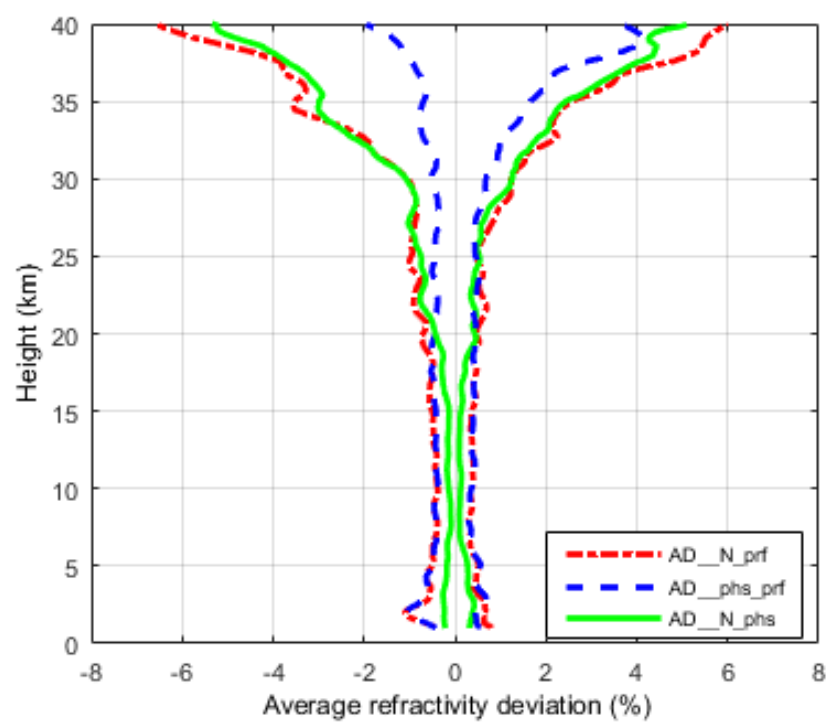

Figure 5. The statistical average relative deviation results of the refractivity for rising occultation events. AD_N_prf denotes the average relative deviation of refractivity between $\mathrm{R} \_\mathrm{N}$ and atmPrf; AD_phs_prf expresses the average relative deviation of refractivity between R_phs and atmPrf; AD_N_phs represents average relative deviation of refractivity between $\mathrm{R} \_\mathrm{N}$ and $\mathrm{R} \_$phs; $\mathrm{R} \_\mathrm{N}$ is the refractivity derived from ND-derived AEP for the selected 200 RO events based on ROPP software; Ref_Phs is the refractivity obtained from atmPhs profiles for the selected $200 \mathrm{RO}$ events based on ROPP software.

we randomly select $200 \mathrm{RO}$ events on 12 December 2013 in order to obtain the AEPm, utilizing Eq. (5). Then we derive profiles of refractivity and dry temperature through ROPP software, which are named R_N and T_N. Figure 3 shows the distribution of the $1605 \mathrm{RO}$ events on 12 December 2013 and the selected $200 \mathrm{RO}$ events (blue triangle).

There are 112 setting occultation events and 88 rising occultation events in the selected 200 occultation events. ROPP software is implemented to process the atmPhs profiles, which are collocated with the selected $200 \mathrm{RO}$ events and derive profiles of refractivity and dry temperature, which are denoted by $\mathrm{R}$ _phs and $\mathrm{T}$ _phs. We then respectively analyse the setting occultation events and rising occultation events to obtain the average relative deviation of refractivity between R_N and R_phs, R_N and atmPrf, R_phs and atmPrf.

As shown in Figs. 4 and 5, the AD_phs_prf are all less than $\pm 1.5 \%$ in setting occultation events and $\pm 4.2 \%$ in rising occultation events, which once again verifies the feasibility of ROPP software. In addition, Figs. 4 and 5 also indicate that AD_N_phs is closer to 0 than AD_phs_prf and AD_N_prf below $20 \mathrm{~km}$, while the AD_phs_prf are closer to 0 than the AD_N_prf and AD_N_phs from 20 to $40 \mathrm{~km}$. Besides, it is also clear that the AD_N_prf and the AD_N_phs gradually increase with height from 20 to $40 \mathrm{~km}$. The main reasons are that (1) the ionospheric error cannot be completely corrected and the receiver tracking offset is difficult to correct above 

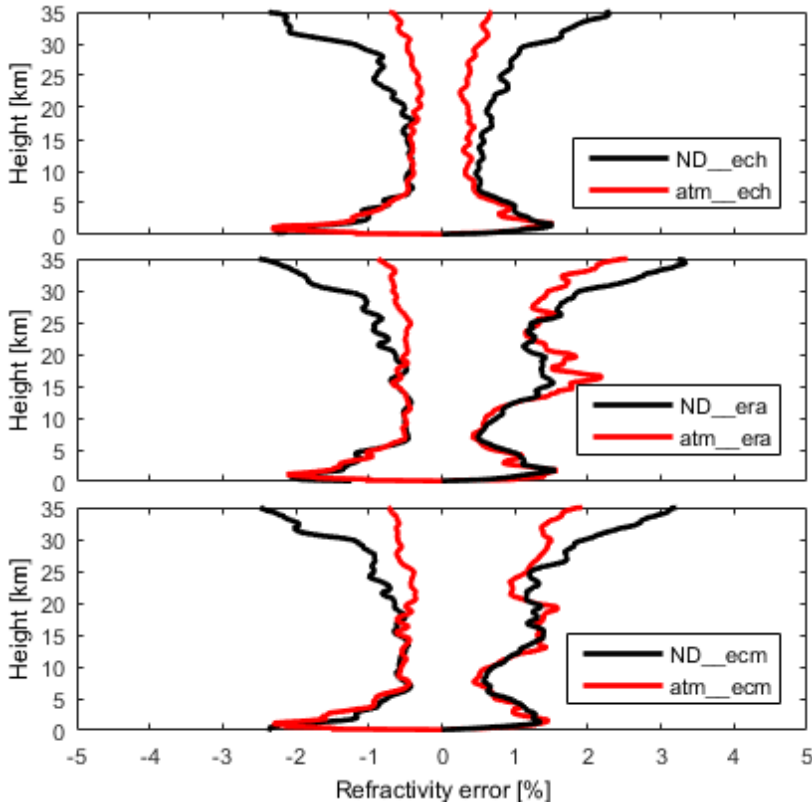

Figure 6. The mean relative refractivity deviation compared R_N and atmPrf with ECMWF analyses in setting RO events. ND_ech represents the average relative refractivity deviation between NDderived and echPrf; atm_ech denotes the average relative refractivity deviation between atmPrf and echPrf; ND_era shows the average relative refractivity deviation between ND-derived and eraPrf; atm_era expresses the average relative refractivity deviation between atmPrf and eraPrf; ND_ecm is the relative refractivity deviation between ND-derived and ecmPrf; atm_ecm signifies the average relative refractivity deviation between atmPrf and ecmPrf; $\mathrm{R} \_\mathrm{N}$ is the relative refractivity derived from ND-derived AEP for the selected 200 RO events based on ROPP software.

$20 \mathrm{~km}$, and (2) due to the occurrence of atmospheric multipath ray propagation in the troposphere, the atmospheric water vapour ambiguity cannot be determined below $10 \mathrm{~km}$. At the same time, statistics on the refractivity and dry temperature difference between ROPP-derived and atmPrf profiles for the selected $200 \mathrm{RO}$ events are listed in Table 4.

Table 4 provides the rms of the comparison between ROPP-derived and atmPrf profiles in the rising and setting $\mathrm{RO}$ events respectively. The statistical results indicate that the accuracy of setting RO events is better than the one in the rising RO events. Besides, the rms of average refractivity differences between ND-derived and atmPrf-derived is better than $2 \%$, and the rms of average dry temperature deviations between ND-derived and atmPrf -derived is better than $3.3 \%$.

\subsection{Comparison with ECMWF}

The CDAAC/UCAR centre provides the ECMWF analysis products, including ecmPrf, echPrf and eraPrf profiles which have collocated with radio occultation profiles. Among them, ecmPrf profiles contain temperature, pressure and moisture

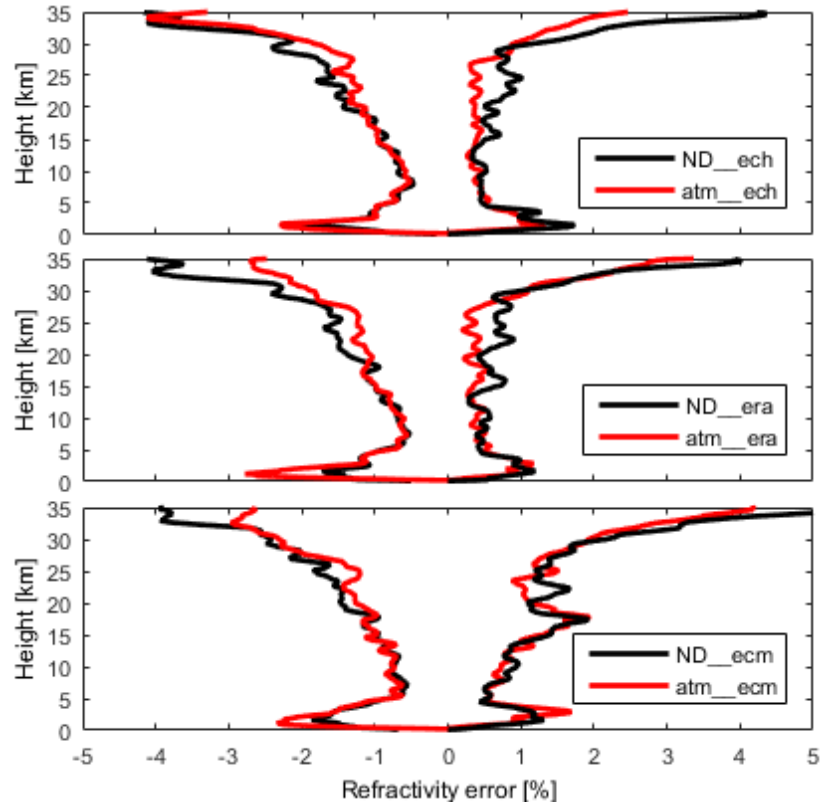

Figure 7. The mean relative refractivity deviation compared R_N and atmPrf with ECMWF analyses in rising RO events. ND_ech represents the average relative refractivity deviation between NDderived and echPrf; atm_ech denotes the average relative refractivity deviation between atmPrf and echPrf; ND_era shows the average relative refractivity deviation between ND-derived and eraPrf; atm_era expresses the average relative refractivity deviation between atmPrf and eraPrf; ND_ecm is the average relative refractivity deviation between ND-derived and ecmPrf; atm_ecm signifies the average relative refractivity deviation between atmPrf and ecmPrf; R_N is the refractivity derived from ND-derived AEP for the selected 200 RO events based on ROPP software.

profiles generated from ECMWF analysis with 21 layers; echPrf profiles contain temperature, pressure and moisture profile from ECMWF high precision analysis field data with 88 layers; and eraPrf profiles include temperature, pressure and moisture profiles generated from the ERA interim model with 37 layers. Then, the COSMIC refractivity profiles observed using the ND method are compared with these three kinds of products. The mean relative refractivity deviations by comparing ND-derived and atmPrf profiles with ECMWF analyses are shown in Fig. 6 for setting RO events and in Fig. 7 for rising RO events.

From Figs. 6 and 7, it can be seen that the mean relative refractivity deviation (MRRD) between $\mathrm{R} \_\mathrm{N}$ and ECMWF analyses is closer to 0 than the MRRD between atmPrf and ECMWF analyses below the height of $10 \mathrm{~km}$, while the MRRD between R_N and ECMWF analyses are closer to 0 than the MRRD between atmPrf and ECMWF analyses from 10 to $35 \mathrm{~km}$. This could be the reason that the non-difference method cuts down the noise level on the excess phase paths and thereby obtains less-biased refractivity within regions of multipath signal propagation in the lower troposphere com- 
Table 5. The summary of the mean relative refractivity deviation between COSMIC observations and ECMWF analyses (\%).

\begin{tabular}{llrrr}
\hline Parameter & Status & ecmPrf & eraPrf & echPrf \\
\hline R_N & rising & 1.82 & 1.46 & 1.49 \\
& setting & 1.32 & 1.34 & 1.06 \\
atmPrf & rising & 1.61 & 1.20 & 1.19 \\
& setting & 0.99 & 1.04 & 0.62 \\
\hline
\end{tabular}

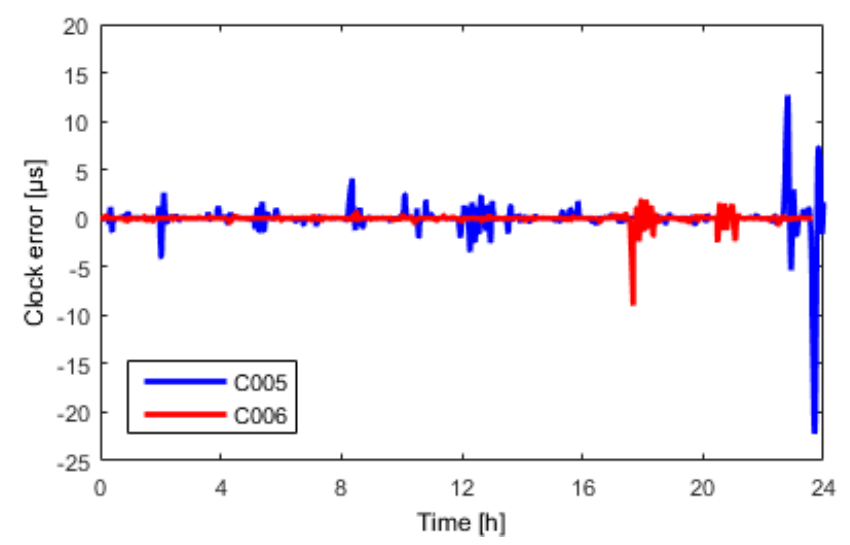

Figure 8. The 5th and 6th COSMIC receiver clock error on 12 December 2013. C005 represents the 5th COSMIC RO satellite; C006 represents the 6th COSMIC RO satellite.

pared to the single-difference technique. However, the multipath is assumed to be negligible and the bending angles obtained from full spectrum inversion (FSI) are replaced by the results derived from the geometric optics above $20 \mathrm{~km}$. The stratosphere refractivity mainly affects the respective ionospheric correction procedure. Moreover, the ND results have a good agreement with SD profile throughout the full altitude range, and the difference between ND and SD results with ECMWF in the lower troposphere is obviously caused by the reference link phase noise. In addition, we also provide the statistical results between R_N and atmPrf and ECMWF analyses in Table 5.

Table 5 indicates that the accuracy of $\mathrm{R} \_\mathrm{N}$ is slightly worse than atmPrf compared to ECMWF analyses data. This is because it is subject to the effect of the COSMIC receiver clock. Figure 8 shows the 5th and 6th COSMIC receiver clock errors on 12 December 2013.

It can be seen that both the 5th and 6th COSMIC satellite receiver clock offsets exhibit very dramatic changes, which suggests that the COSMIC receiver does not have an ultrastable oscillator. Moreover, it can be seen that the maximum clock difference between the adjacent two epochs is over $20 \mathrm{~ms}$ from Fig. 8. Therefore, it will introduce a large amount of noise when interpolating the COSMIC clock offset solutions into the temporal resolution of $20 \mathrm{~ms}$. Then, this noise will spread to the excess Doppler and affect the accuracy of refractivity. In this study, the process of AEP for each COS-
MIC occultation event based on the non-difference technique will be discarded when a larger COSMIC clock oscillator appears at the same time as the RO event.

\section{Conclusions}

This study focuses on the extraction of the AEP using the undifferenced processing strategy. Firstly, the COSMIC POD processing is used to accurately determine the position and velocity of the satellite mass centre and the receiver offset based on the PANDA software. Then, according to the UCAR/CDAAC-provided opnGps profiles, the gravitational delay error, the relativistic effects, the receiver clock error and the phase centre offsets are taken into account. The atmospheric excess phases can be estimated with the help of precise final GPS orbits and transmitter clock offsets from GPS time using a non-difference approach. Finally, the bending angle, refractive and dry temperature profiles are taken from AEP using ROPP software. Next, the refractivity profiles obtained from the non-difference method are validated by using atmPrf profiles. The case study of representative rising and setting occultation events indicate that the relative refractive offset between ND-derived and atmPrf profile is better than $\pm 2 \%$ below $30 \mathrm{~km}$ while the relative refractive offset gradually increases with an altitude of 30 to $40 \mathrm{~km}$. In addition, the average relative refractive deviation of globally distributed 200 events between ND-derived and atmPrf profiles show that the comparison results are changing from \pm 0.5 to $\pm 6 \%$ for setting RO events, and from \pm 0.5 to $\pm 7 \%$ for rising $\mathrm{RO}$ events. The statistical results of refractivity and dry temperature are better than 2.0 and $3.3 \%$ respectively. Finally, the mean relative refractivity deviation between COSMIC observations and ECMWF analyses indicates that the non-difference approach reduces the noise level on the excess phase paths in the lower troposphere when compared to the single-difference processing strategy. Subject to the impact of the receiver clock oscillation, the atmospheric excess phase process may fail using the non-difference processing strategy in partial RO events. If the second-generation COSMIC receiver equipped an ultra-stable oscillator, it will improve the quality of AEP using the ND technique. Not only can the accuracy and the resolution of the LEO POD be improved in the future, but so can the accuracy of refractivity.

Data availability. The Constellation Observing System for Meteorology, Ionosphere and Climate-1 program (CDAAC, 2017) offers the COSMIC radio occultation data, which can be freely obtained from http://cosmic-io.cosmic.ucar.edu/cdaac/index.html.

Competing interests. The authors declare that they have no conflict of interest. 
Acknowledgements. The financial support from National 973 Program of China (no. 2012CB957701), National Natural Science Foundation of China (nos. 41074008), and Key Laboratory of Geospace Environment and Geodesy, Ministry of Education (nos. 16-02-09) is greatly appreciated. We thank UCAR/CDAAC for providing COSMIC data.

Edited by: I. Moradi

Reviewed by: two anonymous referees

\section{References}

Ashby, N.: Relativity in the global positioning system, Living Rev. Relativ., 6, doi:10.12942/lrr-2003-1 , 2003.

Beyerle, G., Schmidt, T., Michalak, G.: GPS radio occultation with GRACE: Atmospheric profiling utilizing the zero difference technique, Geophys. Res. Lett., 32, L1386, doi:10.1029/2005GL023109, 2005.

Bi, Y. M., Chen, J., Yang, G. L., Liao, M., and Wu, R. H.: GPS occultation excess phase computed utilizing the updated single difference technique, Acta Phys. Sin., 61, 149301-149308, 2012.

COSMIC Operations Group: CDAAC Data Products, available at: http://cdaac-www.cosmic.ucar.edu/cdaac/products.html (last access: 10 May 2017), 2013.

COSMIC Data Analysis and Archive Center (CDAAC): COSMIC Radio Occultation data, available at: http://cosmic-io.cosmic. ucar.edu/cdaac/index.html, last access: 10 May 2017.

Esteban, E., Vazquez, B., Borota, A., and Grejner, B.: GPS-PWV estimation and validation with radiosonde data and numerical weather prediction model in Antarctica, GPS Solut., 17, 29-39, 2013.

Hajj, G. A., Kursinski, E. R., Romans, L. J., Bertiger, W. I., and Leroy, S. S.: A technical description of atmospheric sounding by GPS occultation, J. Atmos. Sol. Terr. Phys., 64, 451-469, 2002.

Hwang, C. W., Tseng, T. P., and Lin, T. J.: Precise orbit determination for the FORMOSAT-3/COSMIC satellite mission using GPS, J. Geodesy, 83, 477-489, 2009.

Kuo, B., Rocken, C., and Anthes, R.: GPS radio occultation missions, The second Formosat3/ COSMIC Data Users Workshop, Boulder, Colorado, 2007.

Kursinski, E. R., Hajj, G. A., and Schofield, J. T.: Observing earth's atmosphere with radio occultation measurements using the Global Positioning System, J. Geophys. Res., 102, 2342923465, 1997.

Liu, J. N. and Ge, M. R.: PANDA software and its preliminary result of positioning and orbit determination, Wuhan University Journal of Natural Sciences, 8, 603-609, 2003.

Liu, J. N., Zhao, Q. L., and Ge, M. R.: Preliminary result of CHAMP orbit determination with PANDA software, The International Symposium on GPS/GNSS, Sydney Australia, 2004.

Montenbruck, O., Garcia-Fernandez, M., and Williams, J.: Performance comparison of semi-codeless GPS receivers for LEO satellites, GPS Solut., 10, 249-261, doi:10.1007/s10291-0060025-9, 2006
Rocken, C., Anthes, R., Exner, M., Hunt, D., Sokolovskiy, S., Ware, R., Gorbunov, M., Schreiner, W., Feng, D., Herman, B., Kuo, Y.H., and Zou, X.: Analysis and validation of GPS/MET Data in the neutral atmosphere, J. Geophys. Res., 102, 29849-29866, 1997.

Rocken, C., Kuo, Y. H., and Schreiner, W.: COSMIC system description, special issue of terrestrial, Atmos. Ocean. Sci., 11, 21 52, 2000.

Schreiner, B.: COSMIC GPS POD and limb antenna test report, Internal report of UCAR, 2005.

Schreiner, W. and Rocken, C.: Quality assessment of COSMIC/FORMOSAT-3 GPS radio occultation data derived from single-and double-difference atmospheric excess phase procession, GPS Solut., 14, 13-22, 2010.

Schreiner, W., Rochen, C., and Hunt, D.: Approach and quality assessment of single difference processing of GPS radio occultation data at the UCAR CDAAC, A43A-0066, AGU Fall Meeting, San Francisco, CA, 11-15 December, 2005.

Schreiner, W., Sokolovskiy, S., Hunt, D., Rocken, C., and Kuo, Y.-H.: Analysis of GPS radio occultation data from the FORMOSAT-3/COSMIC and Metop/GRAS missions at CDAAC, Atmos. Meas. Tech., 4, 2255-2272, doi:10.5194/amt4-2255-2011, 2011.

Sokolovskiy, S. and Hunt, D.: Statistical optimization approach for GPS/MET data inversions, presentation at URSI GPS/MET Workshop, Tucson, AZ, 1996.

Ware, R.: GPS sounding of the atmosphere from low earth orbit: Preliminary results, B. Am. Meteorol. Soc., 77, 19-40, 1996.

Wilson, J., Anderson, C., and Baker, M.: Radiometric calibration of the advanced wind scatter meter radar ASCAT carried onboard the METOP-A satellite, IEEE T. Geosci. Remote, 48, 32363255, 2010.

Wickert, J., Reigber, C., Beyerle, G., and Konig, R.: Atmosphere sounding by GPS radio occultation: first results from CHAMP, Geophys. Res. Lett., 28, 3263-3266, 2001.

Wickert, J., Beyerle, G., Hajj, G., Schwieger, V., and Reigber, C.: GPS radio occultation with CHAMP: atmospheric profiling utilizing the space-based single difference technique, Geophys. Res. Lett., 29, 1187, doi:10.1029/2001GL013982, 2002.

Wickert, J., Beyerle, G., König, R., Heise, S., Grunwaldt, L., Michalak, G., Reigber, Ch., and Schmidt, T.: GPS radio occultation with CHAMP and GRACE: A first look at a new and promising satellite configuration for global atmospheric sounding, Ann. Geophys., 23, 653-658, doi:10.5194/angeo-23-653-2005, 2005.

Wu, B. H., Fu, C. L., Liou, Y. A., Chen, W. J., and Pan, H. P.: Quantitative analysis of the errors associated with orbit uncertainty for FORMOSAT-3, in: Proceeding of the international symposium on remote sensing (ISRS), 87-90, 2005.

Yen, N. L. and Huang Chen, J. F.: FORMOSAT-3/COSMIC GPS radio occultation mission: preliminary results, IEEE T. Geosci. Remote, 45, 3813-3825, 2007. 